\title{
Online Test Structure for Measuring Young's Modulus and Residual Stress of the Top Silicon Layer of Silicon-On-Insulator by a Pull-in Approach
}

\author{
Shixuan Gao, Zaifa Zhou*, Weihua Li and Qing-an Huang \\ Key Laboratory of MEMS of the Ministry of Education, Nanjing 210096, China
}

(Received July 14, 2014; accepted December 8, 2014)

Key words: SOI, measurement, pull-in, mechanical properties, in situ

In this study, we proposed a novel test structure that can eliminate the effects of gravity and the release process and compared it with the traditional pull-in structure where the beam can vibrate laterally. This novel structure, which simply uses the top silicon layer to form a complete pull-in test structure, processes both a fixed-fixed beam and a fixed electrode on the top silicon layer of silicon-on-insulator (SOI). In addition, the equation concerning the applied voltage, Young's modulus, and residual stress was developed by the energy method. A parametric simulation was performed to obtain a structure with optimized dimensions and satisfy the equation concerning the applied voltage, Young's modulus, and residual stress. Experimental results show that the measurement system used has the advantages of high precision and rapid testing. The measured average Young's modulus is $110.9 \mathrm{GPa}$ and the residual stress is $4.4914 \mathrm{MPa}$.

\section{Introduction}

Micro-electromechanical systems (MEMS) are cutting-edge technologies developed from integrated circuit (IC) technology. For thin films, the key material properties, e.g., Young's modulus and the residual stress $\sigma$, are very sensitive to the fabrication method.(1) The application of thin films depends on their mechanical properties to a large extent. ${ }^{(2)}$ To provide information regarding material parameters for MEMS engineers both for design and optimization, it is nesseary to extract material properties for a given process. ${ }^{(3-5)}$

The theory of electrostatic pull-in bias has been well documented by Petersen. ${ }^{(6)}$ Afterwards, Najafi and Suzuki, ${ }^{(7)}$ and Osterberg and Senturia have rectified the structure. ${ }^{(8)}$ Sharma and DasGupta have just rectified the method.(4) We must admit that the electrostatic pull-in is a well-known sharp instability in the behavior of an elastically supported structure subjected to parallel-plate electrostatic actuation. ${ }^{(8)}$ It will result in

${ }^{*}$ Corresponding author: e-mail: zfzhou@seu.edu.cn 
a certain deviation because we make numerous hypotheses and simplify the structure, besides the effects of gravity and the release process..$^{(5,9)}$

Silicon-on-insulator (SOI) wafers are precisely engineered multilayer semiconductor structures that provide new functionalities to advanced Si devices. SOI technology offers significant advantages in the design, fabrication, and performance of many semiconductor circuits. ${ }^{(10)}$ For example, SOI can lower the power, has a higher performance, and easily works at low voltages.

In this study, we proposed a novel test structure that can eliminate the effects of gravity and the release process and compared it with the traditional pull-in structure where the beam can vibrate laterally. A parametric simulation was performed to obtain a structure with optimized dimensions and satisfy the equation concerning the applied voltage, Young's modulus and residual stress. Then, several types and different sizes of test structures were designed and tested. By using the expression that links the pull-in voltage and the mechanical properties, which was developed by using the energy method, it is possible to extract both the Young's modulus and residual stress by changing the geometrical parameters of the structure.

\section{Materials and Methods}

\subsection{Measurement principles}

Figure 1 shows the deformation of the basic structure and the coordinate setting. The principle involves the well-known pull-in voltage. The voltage applied to the fixedfixed beam results in a decrease in gap spacing and thus in an increase in the electrostatic force of the beam toward the substrate. ${ }^{(7)}$ At a critical pull-in voltage, the beam becomes unstable and spontaneously collapses to the electrode.(5)

When the beam deflects, an excess stress $\Delta \sigma$ is produced along the beam and remains constant in accordance with the theory of elasticity. The deflection curve can be expressed as ${ }^{(11,12)}$

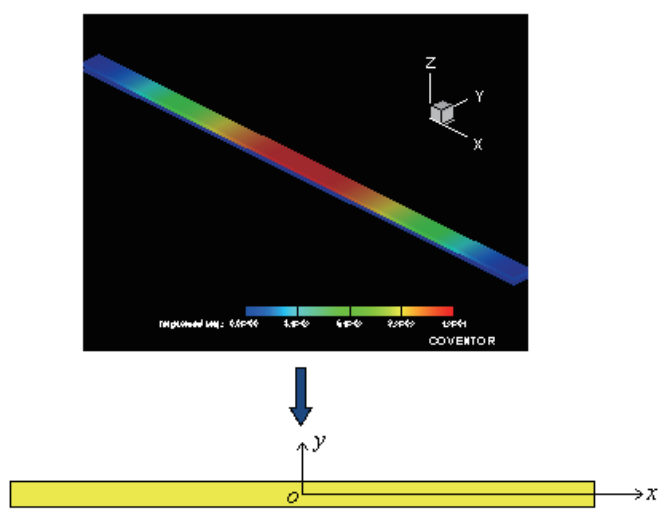

Fig. 1. (Color online) Basic test structure. 


$$
w(x)=\frac{c}{2}\left(1+\cos \frac{2 \pi x}{l}\right) .
$$

The axial deformation energy of the beam, $W_{\mathrm{m}}$, is

$$
W_{\mathrm{m}}=\frac{\sigma^{2} h b l}{2 E}=\frac{\left(\sigma_{x}+\Delta \sigma\right)^{2} h b l}{2 E} .
$$

Here,

$$
\begin{gathered}
\Delta \sigma=\frac{\Delta l}{l}=\frac{1}{2 l} \int_{-\frac{l}{2}}^{\frac{l}{2}}\left(\frac{\mathrm{d} w}{\mathrm{~d} x}\right)^{2}=\frac{\pi^{2} \omega_{1}^{2}}{4 l^{2}}, \\
W_{\mathrm{m}}=\frac{h b l \sigma^{2}}{2 E}+\frac{\pi^{2} h b \sigma \omega_{1}^{2}}{4 l}+\frac{\pi^{4} h b E \omega_{1}^{4}}{32 l^{3}} .
\end{gathered}
$$

The bending energy of the beam, $W_{\mathrm{b}}$, is

$$
W_{\mathrm{b}}=\frac{E I}{2} \int_{-\frac{l}{2}}^{\frac{l}{2}}\left(\frac{\mathrm{d}^{2} w}{\mathrm{~d} x^{2}}\right)^{2} \mathrm{~d} x=\frac{\pi^{4} E c^{2} b h^{3}}{12 l^{3}} .
$$

Lastly, the elastic energy of the beam, $W_{\text {elastic }}$, contains the bending energy of the beam, $W_{\mathrm{b}}$, and the axial deformation energy of the beam, $W_{\mathrm{m}} \cdot{ }^{(13)}$

$$
W_{\text {elastic }}=W_{\mathrm{b}}+W_{\mathrm{m}}=\frac{h b l \sigma^{2}}{2 E}+\frac{\pi^{2} h b \sigma \omega_{1}^{2}}{4 l}+\frac{\pi^{4} h b E \omega_{1}^{4}}{32 l^{3}}+\frac{\pi^{4} E c^{2} b h^{3}}{12 l^{3}} .
$$

The electrostatic energy $W_{\mathrm{e}}^{*(13)}$ between two electrodes with the applied voltage $V$ is

$$
W_{\mathrm{e}}^{*}=\frac{V^{2}}{2} \varepsilon_{0} b \frac{l}{\sqrt{\left(g+h_{0} / \varepsilon_{r 0}-c\right)\left(g+h_{0} / \varepsilon_{r 0}\right)}} .
$$

The total energy of the system, $W$, is the sum of $W_{\text {elastic }}$ and $W_{\mathrm{e}}^{*}$.

$$
W=W_{\text {elastic }}+W_{\mathrm{e}}^{*}
$$

On the basis of the results obtained by the energy method,

$$
\frac{\partial W}{\partial c}=\frac{\partial W_{\text {elastic }}}{\partial c}+\frac{\partial W_{\mathrm{e}}^{*}}{\partial c}=0
$$


Considering the boundary condition, we obtain ${ }^{(8)}$

$$
V_{\mathrm{PI}}=\sqrt{4\left(A c_{\mathrm{PI}}+B c_{\mathrm{PI}}^{3}\right) \cdot \sqrt{g} \cdot\left(g-c_{\mathrm{PI}}\right)^{3 / 2} / \varepsilon_{0} / b /(1+0.42 g / b)},
$$

where

$$
A=2\left[\frac{\tilde{E}}{3} \cdot\left(\frac{\pi}{l}\right)^{4} \cdot \frac{h^{3}}{4}+\frac{\pi^{2}}{4 l^{2}} \cdot \sigma h\right] B=\frac{\pi^{4}}{8 l^{4}} \tilde{E} h .
$$

\subsection{Model validation}

To verify the accuracy of the analytical model, a commercially available finite element method (FEM) package, Conventor, was used for numerical analysis. For such an analysis, Young's modulus and Poison's ratio of the material were chosen to be $116 \mathrm{GPa}$ and 0.27 , respectively. The beam was fixed at both ends, the electrode's face, which faces the beam, is also fixed. Figure 2 shows a schematic of the test structure. The simulation result of the voltage applied to the beam is shown in Fig. 3. It is clearly observed that the pull-in voltage, which was determined using eq. (10), agrees well with the data obtained from the simulation. Table 1 shows the dimensions used in the simulations and verifies that the analytical model is accurate.

\subsection{Sample preparation}

Our main work focuses on the top silicon layer of SOI to form a complete pull-in test structure. In our experiments, the top silicon layer is $5 \mu \mathrm{m}$, the $\mathrm{SiO}_{2}$ layer is $1 \mu \mathrm{m}$, and the substrate is about $600 \mu \mathrm{m}$. The structure needs plasma etching, the required steps of which are shown in Fig. 4.
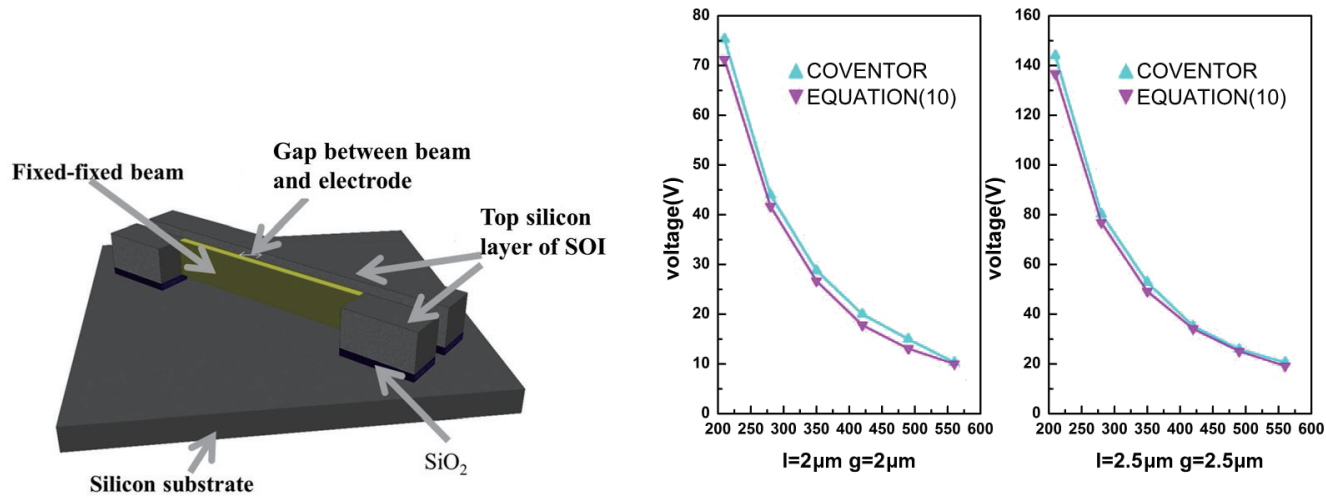

Fig. 2 (left). (Color online) Schematic of test structure.

Fig. 3 (right). (Color online) Pull-in voltage comparison for fixed-fixed beams from Coventor and eq. (10). 
Table 1

Dimensions of the test structures for FEM simulations (in $\mu \mathrm{m}$ ).

\begin{tabular}{lllll}
\hline No. & L & W & H & G \\
\hline 1 & 210 & 5 & 2 & 2 \\
2 & 280 & 5 & 2 & 2 \\
3 & 350 & 5 & 2 & 2 \\
4 & 420 & 5 & 2 & 2 \\
5 & 490 & 5 & 2 & 2 \\
6 & 560 & 5 & 2 & 2 \\
7 & 210 & 5 & 2.5 & 2.5 \\
8 & 280 & 5 & 2.5 & 2.5 \\
9 & 350 & 5 & 2.5 & 2.5 \\
10 & 420 & 5 & 2.5 & 2.5 \\
11 & 490 & 5 & 2.5 & 2.5 \\
12 & 560 & 5 & 2.5 & 2.5 \\
\hline
\end{tabular}
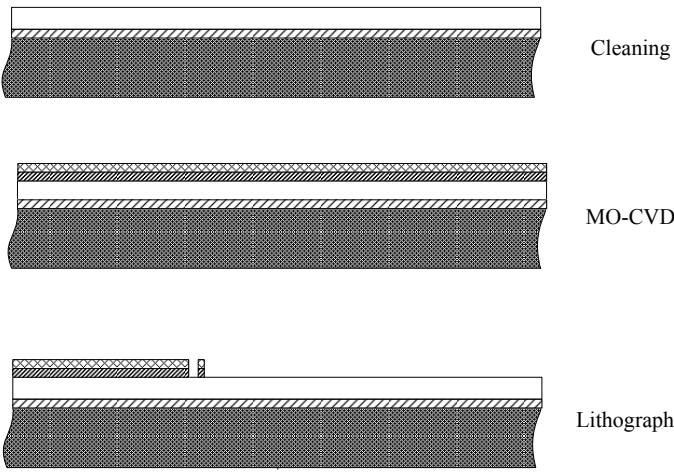

Lithography
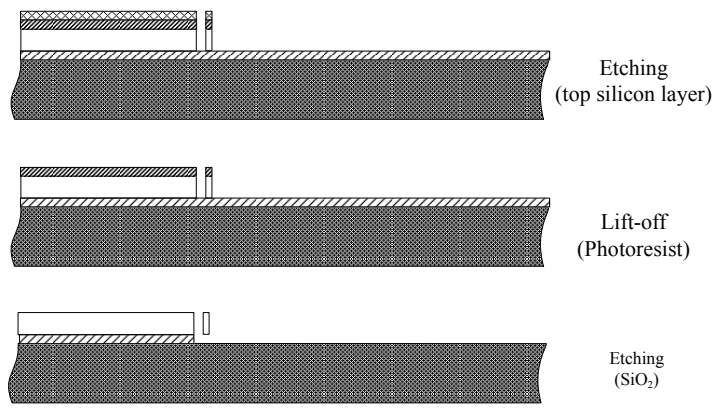

Etching

$\left(\mathrm{SiO}_{2}\right)$

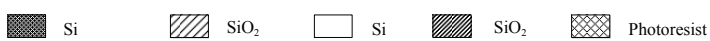

Fig. 4. Sequence of process steps for creating pull-in structures. 
To process both a fixed-fixed beam and a fixed electrode on the top silicon layer of SOI, the $\mathrm{SiO}_{2}$ layer should be etched so that the fixed-fixed beam can vibrate laterally. All of the structures are in arrays, which can be determined by digital holographic microscopy (DHM), showing whether the fixed-fixed beam has residual stress and if it is tensile or compressive. Varying the beam size can optimize the observed residual stress range. Figure 5 shows the scanning electron microscopy (SEM) images of the test structure.

\section{Results}

The next step is the measurement of $V_{\mathrm{PI}}$, as illustrated in Fig. 6(a). In our experiment, $V_{\mathrm{PI}}$ is measured on a probe station. The Keithley device is programmed slowly to ramp

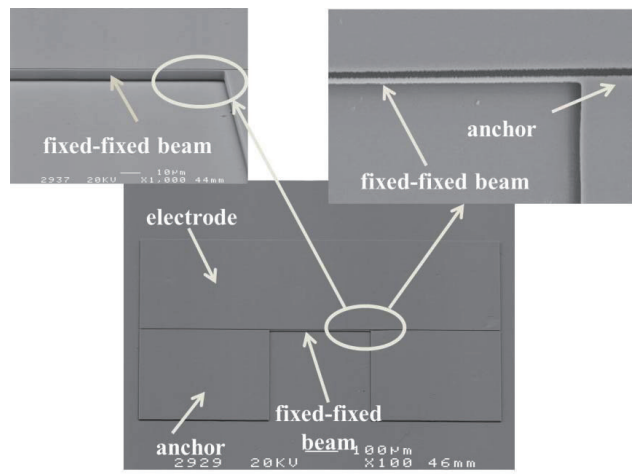

Fig. 5. SEM images of test structure.

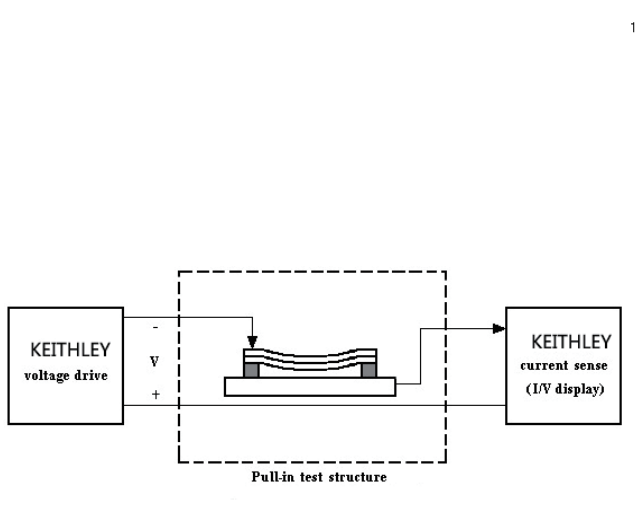

(a)

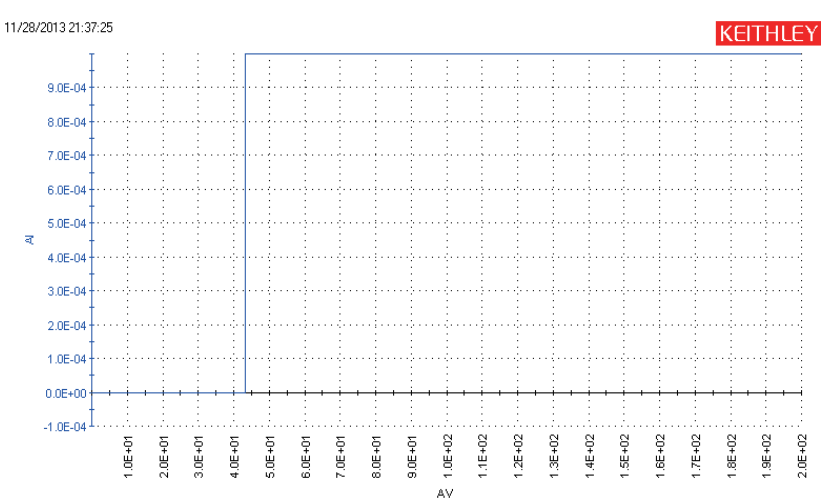

(b)

Fig. 6. (Color online) (a) Pull-in measurement experimental setup. (b) Example of pull-in measurement using $I / V$ output on Keithley. (This example shows the pull-in measurement for a $280-\mu \mathrm{m}$-long beam, 5 - $\mu \mathrm{m}$-wide, $2-\mu \mathrm{m}$-thick, and $2 \mu \mathrm{m}$ gap between the beam and the electrode. The current of the Keithley device is set to $0.1 \mu \mathrm{A}$.) 
Table 2

Results of measurements.

\begin{tabular}{llllll}
\hline No. & L & W & H & G & Pull-in voltage \\
\hline 1 & 280 & 5 & 2 & 2 & 29.4 \\
2 & 280 & 5 & 2 & 2 & 29.6 \\
3 & 280 & 5 & 2 & 2 & 43.4 \\
4 & 280 & 5 & 2 & 2 & 44 \\
5 & 350 & 5 & 2.5 & 2.5 & 52 \\
6 & 350 & 5 & 2.5 & 2.5 & 54 \\
7 & 350 & 5 & 2.5 & 2.5 & 79.5 \\
8 & 350 & 5 & 2.5 & 2.5 & 84 \\
\hline
\end{tabular}

the voltage applied to the beam at $0.1 \mathrm{~V} / \mathrm{s}$, because the speed is slow but it can measure the pull-in voltage more precisely. When the voltage applied to the beam reaches the pull-in voltage, we can see a distinct step on the screen [see the example data in Fig. 6(b)].

The pull-in voltages of the samples are shown in Table 2. From the measured value, we can extract both Young's modulus and residual stress using eq. (10). The Young's modulus is $110.9 \pm 5.02 \mathrm{GPa}$ and the residual stress is $4.4914 \pm 1.000933 \mathrm{MPa}$.

In this study, some approximations and assumptions were made when building the model. However, their effects were negligible. The process of sample fabrication may also affect the test precision.

\section{Conclusions}

In this study, we proposed a novel test structure that can eliminate the effects of gravity and the release process and compared it with the traditional pull-in structure where the beam can vibrate laterally. This novel structure, which simply uses the top silicon layer to form a complete pull-in test structure, processes both a fixed-fixed beam and a fixed electrode on the top silicon layer of SOI. In addition, the equation concerning the applied voltage, Young's modulus, and residual stress was developed by the energy method. A parametric simulation was performed to obtain a structure with optimized dimensions and satisfy the equation concerning the applied voltage, Young's modulus, and residual stress. The experimental results show that the measurement system used has the advantages of high precision and rapid testing. The validation and accuracy of the method were analyzed by FEM simulation and experiment. It is clear that the method plays a potential role in the future of production process lines.

\section{Acknowledgements}

The project was supported by the National Science and Technology Major Project under 2011ZX02507. 


\section{References}

1 V. Lebedev, F. Knöbber and N. Heidrich: Phys. Status Solidi C 9 (2012) 403.

2 H. Y. Liu, Z. F. Zhou and W. H. Li: J. Micromech. Microeng. 22 (2012) 055017.

3 L. Li, J. Gomes and G. Brown: J. Micromech. Microeng. 19 (2009) 125027.

4 J. Sharma and A. DasGupta: J. Micro/Nanolithography, MEMS, and MOEMS 7 (2008) 6.

5 H. M. SedighiI and D. Farhang: Latin Am. J. Solids Struct. 11 (2014) 1078.

6 K. E. Petersen: IEEE Trans. Electron Devices 25 (1978) 1241.

7 K. Najafi and K. Suzuki: Proceedings: An Investigation of Micro Structures, Sensors, Actuators, Machines and Robots (IEEE, Salt Lake City, 1989) pp. 96-97.

8 P. M. Osterberg and S. D. Senturia: J. Microelectromech. S. 6 (1997) 107.

9 Y. C. Hu, W. P. Shih and L. G. De: J. Micromech. Microeng. 17 (2007) 1099.

10 G. K. Celler and S. Cristoloveanu: J. Appl. Phys. 93 (2003) 4955.

11 Q. B. Zou, Z. Li and L. Liu: Sens. Actuators, A 48 (1995) 137.

12 S. D. Senturia: Microsystem Design (Kluwer Academic Publishers, Dordrecht, 2001) p. 249.

13 L. D. Gabbay: Computer Aided Macromodeling for MEMS [D], Ph. D Thesis, MIT, 1998, p. 15. 